\title{
Competitive Influence Maximisation using Voting Dynamics
}

\author{
Sukankana Chakraborty \\ Electronics and Computer Science \\ University of Southampton \\ Southampton, UK \\ sc8n15@ecs.soton.ac.uk
}

\author{
Ananthram Swami \\ CCDC \\ Army Research Lab \\ Adelphi,USA \\ ananthram.swami.civ@mail.mil
}

\author{
Sebastian Stein \\ Electronics and Computer Science \\ University of Southampton \\ Southampton, UK \\ ss2@ecs.soton.ac.uk \\ Geeth de Mel \\ IBM Research \\ Daresbury Laboratory \\ Warrington, UK \\ geeth.demel@uk.ibm.com
}

\author{
Markus Brede \\ Electronics and Computer Science \\ University of Southampton \\ Southampton, UK \\ markus.brede@soton.ac.uk \\ Valerio Restocchi \\ School of Informatics \\ The University of Edinburgh \\ Edinburgh, UK \\ V.Restocchi@ed.ac.uk
}

\begin{abstract}
We identify optimal strategies for maximising influence within a social network in competitive settings under budget constraints. While existing work has focussed on simple threshold models, we consider more realistic settings, where (i) states are dynamic, i.e., nodes oscillate between influenced and uninfluenced states, and (ii) continuous amounts of resources (e.g., incentives or effort) can be expended on the nodes.

We propose a mathematical model using voting dynamics to characterise optimal strategies in a prototypical star topology against known and unknown adversarial strategies. In cases where the adversarial strategy is unknown, we characterise the Nash Equilibrium. To generalise the work further, we introduce a fixed cost incurred to gain access to nodes, together with the dynamic cost proportional to the influence exerted on the nodes, constrained by the same budget. We observe that, as the cost changes, the system interpolates between the historic discrete and the current continuous case.
\end{abstract}

Index Terms-influence maximisation, voting dynamics, game theory, star graphs

\section{INTRODUCTION}

Individual opinions and behaviours are largely affected by peer influence [6]. The propagation of such influence, through societies, predominantly depend on the exchange of information between individuals through their social connections [7]. This inherent connectivity in social networks, can be exploited to fuel several agendas, where societies are externally manipulated through information control, to dominate aggregate social behaviours or steer public opinions, with both positive [25] and negative [14] intent. However, as an external agent trying to influence a social network, the most challenging task, which is

Publication rights licensed to ACM. ACM acknowledges that this contribution was authored or co-authored by an employee, contractor or affiliate of the United States government. As such, the United States Government retains a nonexclusive, royalty-free right to publish or reproduce this article, or to allow others to do so, for Government purposes only.

ASONAM '19, August 27-30, 2019, Vancouver, Canada

(C) 2019 Copyright is held by the owner/author(s). Publication rights licensed to ACM.

ACM 978-1-4503-6868-1/19/08...\$15.00

http://dx.doi.org/10.1145/3341161.3345025 the crux of what is known as the Influence Maximisation (IM) problem [3], is to determine the most optimal distribution of resources that maximises the influence spread.

Traditionally, solutions to this $N P$-hard problem have been offered using approximation algorithms on simple influence models, that guarantee theoretical convergence. The Independent Cascade (IC) model [3] is one such prime example, where influence is introduced with a single injection, which then, in subsequent time steps, cascades throughout the rest of the network. Despite its popularity in $I M$ study, the $I C$ model has its own set of limitations.

Firstly, the IC model only allows a single opportunity to each node, to influence its neighbours. This restricts the scope of its application to many real-world scenarios, where nodes experience external influence from multiple sources and neighbours, at different points in time. Secondly, cascade models assume that nodes in the network are static in nature, i.e. once influenced, they cannot revert back to their original state. While this aptly represents opinions and decisions with long-term commitments, such as buying a car, the irrevocable nature of the nodes makes them equally unsuitable for scenarios where individual choices (or opinions) are transient and free of commitments, such as decisions to intermittently switch between mobile networks for various reasons like cost, affordability and so on [15].

Dynamic models of influence propagation on the other hand, allow nodes in the network to switch from one state to another, thus remedying the drawbacks of static models. Examples include the Voter Model, Majority-Rule Model and the Naming Game [16]. Here, we choose voting dynamics [13] to represent influence propagation in our model. Despite the simplicity of its approach, the voter model has gained substantial popularity in $I M$ study. The most important advantage offered by this model, is that the influence flow equations can be solved analytically for any network, however large. Such analytical solutions are important in their own 


\section{IEEE/ACM International Conference on Advances in Social Networks Analysis and Mining}

right, as they provide benchmarks for computational tools and help understand results in more complex settings. However, analytical solutions typically require an analysis of simplified network structures, such as complete graphs, line graphs, cycles or star graphs. Here, for our work, we deliberately focus on star networks.

Star networks, are not only largely prevalent in social networks, such as in social media (e.g., Twitter) [10] and within organisations [8], but is also an archetypal example of leader-follower structures in social networks. The bimodal degree distribution of star networks creates a trade-off between the roles of low-degree follower nodes and high degree leader nodes, in the influence spread process.

Here, under competitive settings, we determine conditions under which external influence shifts from the hub to the periphery and vice-versa. Contrary to previous $I M$ work [9] [4], which consistently argue the importance of hub nodes over peripheral nodes in the $I M$ process, in this paper, we identify conditions within star networks, where influence is exclusively exerted on the periphery, and not on the hub node. We discuss these results at length, in the latter sections of the paper.

In our given problem, we focus on competitive IM (also interchangeably used with adversarial $I M$ in the rest of the paper), in which two or more contenders compete to maximise their influence within a population. Over the years, competitive $I M$ has attracted considerable attention. A motivating factor is its commercial value in many real-world scenarios, such as viral marketing, where firms compete with one another in oligopolistic markets to maximise the consumption of their own products [1], or in politics, where parties compete with one another to increase their own influence in the population, to win elections [11].

Competitive $I M$ was first proposed as extensions [1] [2] to the IC model [3]. This spurred a fairly large body of work that studied competitive $I M$ using different models and under various social conditions [17] [18] [19]. In dynamics models it has been studied using zealotry [9] [12]. Zealots are agents with extremist opinions (often termed as stubborn agents), which do not change their states, and further, unidirectionally influence other agents in its social neighbourhood to adopt the opinion they endorse.

Despite the rich available background in $I M$ literature, we find that most historic work in this field is characterised by the use of discrete models where nodes are either influenced or not. In contrast, our work here considers a continuous distribution of influence on the network where a broad spectrum of nodes are targeted with varying amounts of influence. Moreover, work in competitive $I M$ largely studies networks that have already been seeded by a competitor. This requires prior knowledge of adversarial strategies which may not always be readily available. In cases where adversarial strategies are unknown, game-theory provides a viable approach to determine the optimal solution to the $I M$ problem.

While there is an abundance of work in game-theoretic $I M$ using static models [23] [24], dynamic models have received limited attention in this aspect. Exceptions are [20] [21] [22], of which, we find that work in [22] bears closest resemblance to our model. The authors study competitive $I M$ using a game-theoretic approach in [22], while assuming that influence spread follows voting dynamics. They however, inject external influence in the network in a single time-step, which starkly contrasts the continuous nature of our influence distribution.

In the following sections of the paper, we study competitive $I M$ in star networks, under various known and unknown adversarial conditions, using the dynamic voter model characterised by continuous allocation of resources. We find that, in case of known adversarial strategies, the best response strategy, when competing influence on the network exceeds available budget, is to target nodes avoided by the adversary with more influence. As available budget increases, more influence is expended on nodes targeted by the adversary. Where adversarial strategy is unknown, we show that the pure-strategy Nash Equilibrium is when both competitors target all nodes equally. We also observe that the optimal strategies change when a fixed cost $c$ is incurred to gain access to nodes in the network. As $c$ increases, the system interpolates between the continuous and discrete models. Where the adversary targets the hub, we find that with increase in cost $c$, the optimal strategy switches between targeting all nodes, to targeting only the hub node very abruptly. In all other cases of known adversarial strategies, we observe a less abrupt shift in the optimal strategy.

\section{SPREAD DyNAMiCS}

We represent social networks as graphs $G(V, E)$ of vertices $(V)$ and edges $(E)$, where vertices $(V)$ indicate social agents and edges $(E)$, the relationships between them. The strength of these connections are given by the weight adjacency matrix $W$ of the graph $G$, where the $i j^{t h}$ entry $w_{i j}$, gives the strength of the influence, an agent $j$ has on $i$. Here, agents $i, j \in V$ and diagonal elements $w_{i i}=0$ for all agents $\forall i \in V$.

We assume that two external influencers, $A$ and $B$ compete to maximise their influence in the network. In the marketing context, this could be firms competing to maximise the consumption of their products. We consider a stochastic binary model where only two states $A$ and $B$ are available to the agents in the network. The state $\sigma_{i} \in\{A, B\}$ of an agent $i \in V$, indicates its affiliation to one of the two competing influences. Here at the beginning of the influence spreading process $(t=0)$, every agent $i$, in the network is assumed to inherently have an initial state $\sigma_{i} \in\{A, B\}$. This state changes dynamically over time as influence flows through the network.

Influence propagation in this model follows voting dynamics [13], where at each update event, a node $i$ is selected at random (with probability $\frac{1}{|V|}$ ), which then either adopts the state of a randomly (with probability $\frac{1}{\operatorname{deg}(i)}$ ) chosen neighbour $j$ or that of an external influencer $A$ or $B$, when directly influenced.

As per the stated problem, the objective of each influencer ( $A$ and $B$ ) is to maximise the number of nodes in the network that adopt their endorsed opinion, at steady-state (after which, fractions of opinions in the network no longer change with time). The probabilistic state of a node $i$ is given by $u_{A, i}$. 


\section{IEEE/ACM International Conference on Advances in Social Networks Analysis and Mining}

This is further defined as the probability with which a node $i$ adopts the state $\sigma_{i}=A$. At equilibrium, the fraction of the total nodes in any one particular state, say $\sigma_{i}=A$, is given by $u_{A, a v g}=\frac{\sum_{i}^{N} u_{A, i}}{N}$. When represented as a non-cooperative game, each player here is driven by the intent to increase their individual pay-off $\mathrm{s} u_{A, \text { avg }}=\frac{\sum_{i}^{N} u_{A, i}}{N}$ and $u_{B, a v g}=\frac{\sum_{i}^{N} u_{B, i}}{N}$.

For any arbitrary network of $N$ nodes, the rate at which an influencer $A$ spreads its influence in the network, is given by:

$$
\frac{d u_{A, i}}{d t}=\left(1-u_{A, i}\right) \cdot p r_{i}(A)-u_{A, i} \cdot p r_{i}(B) .
$$

such that

$$
\begin{array}{r}
\operatorname{pr}_{i}(A)=\frac{\sum_{j} w_{j i} u_{A, j}+p_{A, i}}{\sum_{j} w_{j i}+p_{A, i}+p_{B, i}}, \\
\operatorname{pr}_{i}(B)=\frac{\sum_{j} w_{j i}\left(1-u_{A, j}\right)+p_{B, i}}{\sum_{j} w_{j i}+p_{A, i}+p_{B, i}},
\end{array}
$$

where $\operatorname{pr}_{i}(A)$ is the probability with which a node $i$ adopts state $A$ and $\operatorname{pr}_{i}(B)$ the probability with which it adopts state $B$. Note that, the amounts of influence $p_{A, i}$ and $p_{B, i}$, exerted on any node $i$ here is continuous and proportional to the amount of resource expended on it. Hence, we use resource and influence inter-changeably throughout the course of the paper.

When the system reaches steady-state, although individual states are still dynamic and change with time, the total number nodes in any one particular state in the network, no longer change with time and therefore $\frac{d u_{A, i}}{d t}=0$, gives us

$$
\left[L+\operatorname{diag}\left(\boldsymbol{p}_{\boldsymbol{A}}+\boldsymbol{p}_{\boldsymbol{B}}\right)\right] \boldsymbol{u}_{\boldsymbol{A}}=\boldsymbol{p}_{\boldsymbol{A}} .
$$

Here $L$, the Laplacian of the network is given by the $N-b y-N$ matrix, where each element $L_{i j}=\delta_{i j} \sum_{k} w_{k i}-\left(1-\delta_{i j}\right) w_{j i}$, and $\delta_{i j}$ is the kronecker delta.

While each element of vector $\boldsymbol{u}_{\boldsymbol{A}}$ represents the probability $u_{A, i}$ with which a node $i$ in the network adopts opinion $A$. The sum of its elements divided by to total number of nodes in the network $(N), u_{A, a v g}=\frac{1}{N} \sum_{i=1}^{N} u_{A, i}$, denotes the fraction of total nodes in the network that are in state $A$ (i.e. $\sigma_{i}=A$ ).

Vector $\boldsymbol{p}_{\boldsymbol{A}}$ indicates the distribution of resources $a$, over the network, for an adversary $A$, such that $\sum_{i=1}^{N} p_{A, i}=a$. Here, the elements of each distribution vector $\boldsymbol{p}_{A}$ and $\boldsymbol{p}_{B}$ are non-negative, and $\sum_{i=1}^{N} p_{B, i}=b$.

The objective of this problem is now simply to determine the best possible allocation of resources $\boldsymbol{p}_{\boldsymbol{A}}$, given a fixed $a$, that maximises average opinion $u_{A, a v g}$ in the network. In the following sections, we explore this problem for multiple adversarial settings in star networks. In principle, this method can be extended to other simple networks as well, such as, complete graphs, line graphs, cycles and even small scalefree networks, for which $L$ can be expressed analytically. For arbitrary weighted graphs, the optimisation problem can be solved numerically using 2 , given a fixed budget, $a$ and $b$.

\section{KNOWN ADVERSARIAL STRATEGIES}

In the following sections, we study the competitive IM problem for various known adversarial strategies, with the aim of identifying the best response in each case. We do so in star networks of $N$ nodes, which comprise of two kinds of nodes, distinguishable by their topological positions, i) peripheral (follower) nodes, and ii) a hub (leader) node.

As stated before, adversaries $A$ and $B$ are each assumed to have a certain amount of resources $a$ and $b$ at their disposal, which they allocate to nodes in the network with the intent of influencing them. The best response strategy is the optimal split of resources between the hub and the periphery that results in maximum influence spread in the network. For ease of exposition, we determine optimal strategies only with respect to $A$, against a fixed adversary $B$. We parameterise $A$ 's strategy space using $\alpha$ and $k_{A}$, where $\alpha$ is the amount of resource expended on each peripheral node and $k_{A}$ is the number of peripheral nodes targeted by $A$.

On examining Eq. 2, we find some general cases to explore, where adversarial influence can either be $i$ ) constant, or $i i$ ) proportional to the degree of the node. We study these cases in the following sections.

\section{A. Adversary targets uniformly}

Lemma III.1. The best response strategy against an adversary targeting all nodes in the network uniformly, is also to target all nodes equally.

Proof. We first determine the Laplacian $(L)$ of the star graph with $N$ nodes. This entity does not change as long as the graph structure remains the same. We also define an arbitrary $\mathrm{N}$ dimensional vector $\boldsymbol{p}_{\boldsymbol{A}}$ that represents the resource allocation strategy adopted by $A$. The elements of $\boldsymbol{p}_{\boldsymbol{A}}$ are given by

$$
\boldsymbol{p}_{\boldsymbol{A}, \boldsymbol{i}}= \begin{cases}a-k_{A} \alpha, & \text { for } i=0 \\ \alpha, & \text { for } 1 \leq i \leq k_{A} \\ 0, & \text { for } k_{A}+1 \leq i \leq n\end{cases}
$$

Without loss of generality, here we index the hub node as $i=0$, targeted peripheral nodes as $1 \leq i \leq k_{A}$ and the remaining peripheral nodes as $k_{A}+1 \leq i \leq n$. These notations are maintained throughout the rest of the paper.

Here, vector $\boldsymbol{p}_{B}$ is defined using prior knowledge of adversarial strategy. In this case, where adversary targets all nodes uniformly, it is given by, $p_{B, i}=\frac{b}{n+1}, \forall i \in 0,1 \ldots n$.

Replacing $L, \boldsymbol{p}_{A}$ and $\boldsymbol{p}_{B}$ in Eq. 2] we obtain a system of $N$ non-linear equations, which we solve to get an expression for the average opinion in the network $u_{A, \text { avg }}$, as a function of $k_{A}$ and $\alpha$, given by $u_{A, a v g}=f\left(k_{A}, \alpha\right)$. This allows us to differentiate the expression $u_{A, a v g}$ wrt to the independent variables $k_{A}$ and $\alpha$. We further equate them to 0 , i.e. $\frac{\partial u_{A, a v g}}{\partial k_{A}}=0$ and $\frac{\partial u_{A, a v g}}{\partial \alpha}=0$, and consequently solve them to determine the values $k_{A}^{*}$ and $\alpha^{*}$, for which $u_{A, \text { avg }}$ is maximum. We find that in the case where the adversary targets all nodes uniformly, the best response strategy given by the optimal values $k_{A}^{*}=n$ and $\alpha^{*}=\frac{a}{n+1}$, i.e. to distribute the available budget over all nodes equally. 


\section{IEEE/ACM International Conference on Advances in Social Networks Analysis and Mining}

Note, that this strategy is independent of the total amount of influence $b$ exerted by the adversary on the network, which is a surprising result in a competitive network, as one would expect the response strategy to be affected by change in the strength of adversarial influence. We further observe, that in a star network, where all nodes experience equal amounts of adversarial influence, the degree of the node or its topological position has no bearing on its role in the influence spread process.

The following sections study cases where adversarial influence in the network is proportional to the degree of the node.

\section{B. Adversary targets hub}

Lemma III.2. The best response strategy to an adversary only targeting the hub node is to target all $n$ peripheral nodes, each with $\alpha^{*}=\frac{a+b}{\sqrt{a+b+(n+1)^{2}}+(n+1)}$ and the hub node with the remaining $\left[a-n \alpha^{*}\right]$, for $a \geq b$.

Proof. Here $\boldsymbol{p}_{B}$ is an $N$-vector where $p_{B, 0}=b$ and all other elements $p_{B, i}=0, \forall i \in 1 . . n$. The Laplacian $L$ of the network remains unchanged and $\boldsymbol{p}_{\boldsymbol{A}}$ is defined as before. Using similar methods of differentiation as in the earlier section, we determine the best response strategy as $k_{A}^{*}=n$ and $\alpha^{*}=\frac{a+b}{\sqrt{a+b+(n+1)^{2}}+(n+1)}$.

We observe,

1) Contrary to the case where adversary $B$ targets all nodes uniformly, the best response strategy here, is no longer independent of the strength of the influence $b$ exerted by the adversary on the network.

2) Similar to results obtained in the previous section, the best response strategy here too, is to target all peripheral nodes, $k_{A}^{*}=n$ for $a \geq b$. However, it is important to note that while the optimal strategy against an adversary targeting all nodes uniformly, is to target all nodes equally. Here we see a disparity in the best response strategy, between the amounts of influence given to the hub and the periphery. Furthermore, we note that the distribution of resources between the hub and the periphery strictly depends on the strength of adversarial influence on the network.

3) When adversarial influence $b$ is:

i) less than or equal to $a-$ the best response strategy suggests an uneven split of resources between the hub and the periphery, where the hub receives more influence than peripheral nodes, as $\alpha^{*}=$ $\frac{a+b}{\sqrt{a+b+(n+1)^{2}}+(n+1)}<\frac{a}{n+1}$. Unlike in the earlier case, where all nodes receive the same amounts of influence given by $\alpha^{*}=\frac{a}{n+1}$, here we see that the degree of a node plays an important role in deciding the significance of the node in the influence spread process.

ii) exceeds $a-$ the best response strategy is to only target the periphery and distribute the resources equally over all peripheral nodes. Note that in this case, no resource is expended on the hub. This emerges as an interesting observation, which suggests that as the adversary increases its influence on the hub node, such that $b>a$, no competing influence should be exerted on the hub node itself, or the nodes targeted by the adversary. Instead, all the resources should be expended on the periphery, or nodes avoided by the adversary. This starkly contrasts previous work that emphasise the importance of hub nodes in the $I M$ process [4] [9].

Fig. 1 further provides an illustration of how the optimal strategy switches between $(i)$ targeting the hub, $(i i)$ targeting the periphery, and (iii) targeting all nodes equally, as resources $a$ and $b$ change. For example, we see that for low values of $b$ and high values of $a$, the optimal strategy is to target the hub node with nearly all resources.

\section{Adversary targets periphery}

Lemma III.3. In the case where an adversary B targets only the peripheral nodes, the optimal response strategy is to target all peripheral nodes with $\alpha^{*}=\frac{a+(n+1)-\sqrt{a+b+(n+1)^{2}}}{n}$ and the hub node with the residual $\left[a-n \alpha^{*}\right]$.

Proof. Here, as $B$ only targets the peripheral nodes, the resulting $N$-vector $\boldsymbol{p}_{B}$ is given by $p_{B .0}=0$ and all other elements $p_{B, i}=\frac{b}{n}, i=1$..n. From Eq. 2, we get a system of $N$ non-linear equations, solving which, as before, we get an expression for $u_{A, \text { avg }}=f\left(k_{A}, \alpha\right)$. This can now be differentiated to determine the optimal solutions for $k_{A}$ and $\alpha$, which emerge as $k_{A}^{*}=n$ and $\alpha^{*}=\frac{a+(n+1)-\sqrt{a+b+(n+1)^{2}}}{n}$.

Here too, we observe a non-uniform distribution of resources between the hub and the periphery. Further, akin to results discussed in Sec III-B, where the best response strategy is to spend more resources on the periphery as adversarial influence on the hub increases. Here, we observe that as influence $b$ increases on the network, the value of $\alpha^{*}$ decreases. Therefore, suggesting that as $B$ increases its influence on the periphery, $A$ should devote a greater fraction of its resources on the hub i.e. nodes not targeted by the adversary.

In summary, from this section we conclude and corroborate that, as adversarial influence increases on targeted nodes, the optimal strategy suggests less allocation of resources to these nodes and more to nodes avoided by the adversary.

However, as earlier explained, in certain situations, prior knowledge of adversarial strategy can be an unrealistic assumption. Therefore, to address this limitation, we study cases where adversarial strategies are unknown using a gametheoretic approach. Here, we identify the Nash Equilibrium $(N E)$ (pure or mixed) and further argue its eligibility as the optimal strategy.

\section{GAME-THEORETIC APPROACH}

We define the current problem in the scope of a noncooperative game, where each competitor attempts to maximise their pay-offs by maximising influence in the network, 
2019 IEEE/ACM International Conference on Advances in Social Networks Analysis and Mining
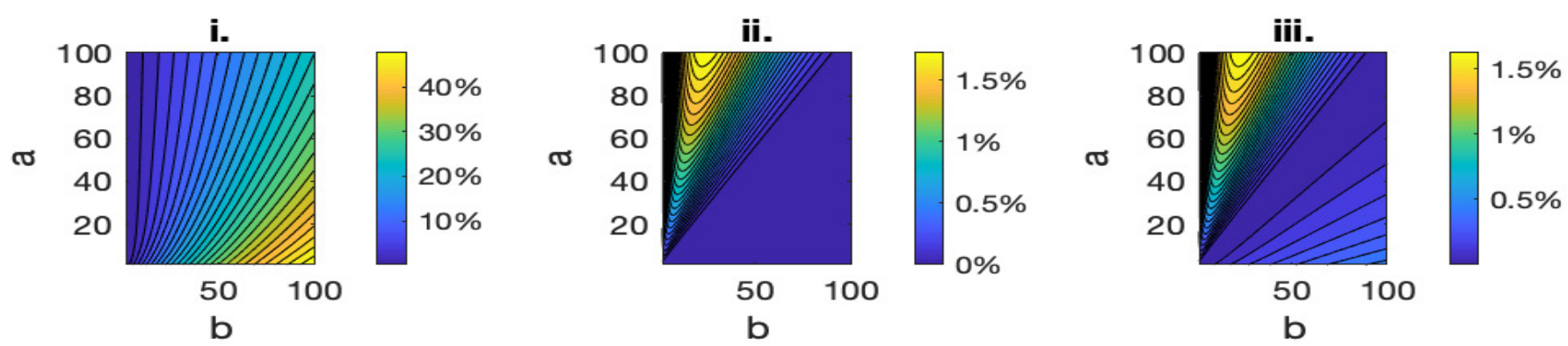

Fig. 1: Heatmaps showing the $\%$ gain in $u_{A, a v g}$, when $A$ plays the optimal strategy given in Lemma III-B in comparison to (i) targeting the hub node, (ii) targeting the periphery and (iii) targeting all nodes uniformly, against adversary $B$ who targets the hub node, for different values of available resources $a$ and $b$, on a star network of $n=100$ nodes.

against an adversary striving to do the same. Note that, although both players are aware of the adversarial strategy space, neither of them have any knowledge about the specific strategy played by the adversary in the current game. Hence, under such circumstances, we are motivated to characterise the $N E$, which we argue is the overall best response strategy. Using game-theoretic terms, the $N E$ in a non-cooperative game with two players is given by the equilibrium strategy from which neither player has the incentive to unilaterally defect.

In the current adversarial $I M$ problem, an $N E$ is characterised in the form of strategies, defined by $\left\{k_{A}^{*}, \alpha^{*}\right\}$ and $\left\{k_{B}^{*}, \beta^{*}\right\}$ for both adversaries, $A$ and $B$.

Here, given the continuous nature of influence distribution, we define the mixed strategy space using $\left\{k_{A}, \alpha\right\}$ and $\left\{k_{B}, \beta\right\}$, and we measure the pay-offs for each player in terms of their average opinions $u_{A, a v g}$ and $u_{B, a v g}$ in the network.

Theorem IV.1. In a star network of $n$ peripheral nodes, the pure strategy $N E$ is at $\left[\left(\frac{a}{n+1}, n\right),\left(\frac{b}{n+1}, n\right)\right]$.

Proof. We define arbitrary vectors for both $\boldsymbol{p}_{\boldsymbol{A}}$ and $\boldsymbol{p}_{B}$ where $k_{A}$ peripheral nodes are targeted with $\alpha$ and $k_{B}$ nodes are targeted with $\beta$ by adversary $B$. The remaining resource in each case is expended on the hub such that $p_{A, 0}=\left(a-k_{A}^{*} \alpha^{*}\right)$ and $p_{B, 0}=\left(b-k_{B}^{*} \beta^{*}\right)$.

The average opinions $u_{A, a v g}$ and $u_{B, a v g}$ in the system are now a result of the individual opinion states of the hub and peripheral nodes of which,

i) $\frac{k_{A} k_{B}}{n}$ are targeted both by $A$ and $B$,

ii) $\left(k_{A}-\frac{k_{A} k_{B}}{n}\right)$ are targeted only by $A$,

iii) $\left(k_{B}-\frac{k_{A} k_{B}}{n}\right)$ are targeted only by $B$ and

iv) $n-\left(k_{A}+k_{B}-\frac{k_{A} k_{B}}{n}\right)$ which are targeted by neither.

We derive both $u_{A, a v g}$ and $u_{B, a v g}$, each from a set of $N$ non-linear equations using $L, \boldsymbol{p}_{A}$ and $\boldsymbol{p}_{B}$ in Eq. 2 These expressions in $u_{A, \text { avg }}=f\left(k_{A}, \alpha\right)$ and $u_{B, \text { avg }}=f\left(k_{B}, \beta\right)$, are then differentiated and solved simultaneously to determine $\left\{k_{A}^{*}, \alpha^{*}\right\}$ and $\left\{k_{B}^{*}, \beta^{*}\right\}$, which serves as the pure-strategy $N E$.

We prove the uniqueness of the equilibrium by first showing that $u_{A, \text { avg }}$ a monotonically increasing in $k_{A}$, i.e. $\frac{\partial u_{A, a v g}}{\partial k_{A}}>0$. Similar can be proven for $u_{B, a v g}$ and $k_{B}$. Now, eliminating all dominated strategies (where $k_{A}<n$ and $k_{B}<n$ ), we solve for $\alpha$ and $\beta$ simultaneously. Here we obtain two solutions, of which only one satisfies the boundary conditions, and is characterised as the pure-strategy $N E$.

Finally, in conclusion, we state that the equilibrium strategy against unknown adversarial strategies is always to target all nodes equally.

\section{Cost Analysis}

We recognise that targeting all nodes may not always be the most feasible strategy. Here, we take a more practical approach and in the process also generalise the model further, by introducing a fixed cost $c$, which we assume is incurred while gaining access to each target node. This cost $c$ in addition to the cost of influence $\alpha$ is constrained by the budget $a$.

When $c=0$ we obtain the optimal solutions enumerated in Sec. III and IV In all cases of known and unknown adversarial strategies we observe that the optimal response is to target all peripheral nodes. However, as $c$ increases, it becomes more expensive to target all nodes in the network as every additional target node constrains the budget even further. As $c$ increases we observe our optimal solutions to resemble those in discrete models [4] [9] . Therefore bridging the gap between traditional discrete models and our continuous model.

In the following sections, we use the same case studies as in Sec. III and IV to compare and observe how optimal strategies change when there is a cost of access $c$ involved.

\section{A. Adversary targets uniformly}

Lemma V.1. The best response strategy against an adversary targeting all nodes uniformly is to target $k_{A}^{*}=$ $-\frac{\alpha(\beta+n+1)+(c-a)(\beta+1)}{\alpha(\beta+\alpha)}$ peripheral nodes, each with $\alpha^{*}=$ $\sqrt{c(\beta+1)}$, where $c$ is a fixed cost paid to gain access to each target node.

Proof. To prove this, we follow the same method used in earlier analyses where $L$ remains unchanged, and $p_{B}$ is the same as in Sec. III-B i.e., $p_{B, i}=\frac{b}{n+1} \forall i \in 0 . . n$. Note that, here $\boldsymbol{p}_{B}$ does not incorporate any cost $c$. This is because, any cost incurred by $B$ can be accounted for, simply by deducting 


\section{IEEE/ACM International Conference on Advances in Social Networks Analysis and Mining}

it from the available budget, to a lower budget. Here, $p_{A, i}$ is defined as

$$
\boldsymbol{p}_{A, \boldsymbol{i}}= \begin{cases}a-k_{A} \alpha-c\left(k_{A}+1\right), & \text { for } i=0 \\ \alpha, & \text { for } 1 \leq i \leq k_{A} \\ 0, & \text { for } k_{A}+1 \leq i \leq n\end{cases}
$$

Replacing the above variables in Eq. 2, we derive $u_{A, \text { avg }}=$ $f\left(k_{A}, \alpha\right)$, which when differentiated and solved simultaneously, gives two solutions for $\alpha^{*}$, only one of which, $\alpha^{*}=\sqrt{c(\beta+1)}$, is positive and hence feasible. This $\alpha^{*}$ yields two solutions for $k_{A}^{*}$. While the solution $k_{A}^{*}=$ $-\frac{\alpha(\beta+n+1)+(c-a)(\beta+1)}{\alpha(\beta+\alpha)}$ is chosen as the optimal value of $k_{A}$, the other solution $k_{A}^{*}=\frac{\alpha(\beta+n+1)+(\beta+1)(a+2(n+1))}{\alpha(\alpha+\beta+2)}$ is discarded, as it defies the budget constraint $c . k_{A} * \leq a$ for all values of $c$. For instance, $k_{A} * \neq 0$ even for very large values of $c$, i.e. $c \approx a$. It is therefore rejected as a viable solution, as $k_{A} *=0$ must be true for large values of $c$.

Here we observe that,

1) With change in the cost of accessing nodes $c$, our optimal strategy interpolates between solutions obtained in the discrete model [4] [9] and the continuous case (Sec. III-B).

i) For small values of $c \approx 0$ the optimal solution targets all peripheral nodes $k_{A}^{*}=n$ (as shown in Sec. III-B. However, when $c$ reaches $c_{n}=\frac{-1}{n}\left[\left(\frac{\eta-\zeta}{2}\right)\left(1+\frac{1}{n}\right)-a\right]$, where $\eta=$ $\sqrt{(n \beta+\beta+n+1)^{2}+4 a n(\beta+1)}$ and $\zeta=(n+$ $1)(\beta+1)$, the value of $k_{A}^{*}$ starts decreasing steadily from $k_{A}^{*}=n$.

ii) As cost $c$ further increases from $c_{n}$, the value of $k_{A}^{*}$ decreases even more, until it reaches $k_{A}^{*}=0$ at cost $c_{0}=\frac{(1+\beta) a^{2}}{(\beta+n)(\beta+n+2)+1}$. At this point, the best response strategy no longer targets any peripheral nodes, resembling results obtained in discrete models [4] [9].

\section{B. Adversary targets hub}

Lemma V.2. The best response strategy when the adversary only targets the hub node, abruptly changes from targeting all nodes to targeting only the hub, when cost of access reaches a critical value $c_{c}=-\frac{2 \chi(n+1)-\varphi}{n+2}$, where $\chi=$ $\frac{1}{n+2}\left(\sqrt{(a+b+n)(n+2)+1}+(n+1)^{2}\right)$ and $\varphi=(a+$ $\left.b+2(n+1)^{2}\right)$.

Proof. First, we substitute the terms $L, \boldsymbol{p}_{\boldsymbol{A}}$ and $\boldsymbol{p}_{\boldsymbol{B}}$ in Eq. 2 Here $\boldsymbol{p}_{\boldsymbol{A}}$ and $L$, have the same values as in Sec V-A while $\boldsymbol{p}_{\boldsymbol{B}}$ is defined as in Sec III-B We now use the same method as before to derive $u_{A, a v g}$ and then differentiate it wrt $k_{A}$ and $\alpha$, to determine their optimal values $k_{A}^{*}$ and $\alpha^{*}$.

We first solve $\frac{\partial u_{A, a v g}}{\partial \alpha}=0$, which gives us two solutions for $\alpha^{*}$ only one of which, is positive and is the acceptable solution, $\alpha^{*}=\frac{\sqrt{\phi+\rho+\gamma}-(n+1)}{k-n-1}$ where $\phi=(a+b-c+n+$ 1) $(n+1), \rho=c k(k-n), \gamma=k(a+b)$. Now replacing $\alpha^{*}$ in $\frac{\partial u_{A, a v g}}{\partial k_{A}}=0$ we solve for $k_{A}^{*}$. This further gives us two solutions, $k_{A}^{*}=(n+1)$ and $k_{A}^{*}=\frac{a+b-c+n+1}{c-1}$ and we validate both solutions for their feasibility in the region $c \in[0, a)$.

So far, we have established that, for a certain value of $c$, particularly when $c \approx 0$, optimal value of $k_{A}^{*}$ should be $k_{A}^{*} \approx$ $n$. However, given the budget constraint $a$, at very high values of $c_{0}$ (such that $c \approx a$ ), $k_{A}^{*}$ must also reduce to $k_{A}^{*}=0$. Our task here is to determine the values of cost $c$ where $k_{A}^{*}=n$, $n \geq k_{A}^{*} \geq 0$ and $k_{A}^{*}=0$ are true.

First, we explore the solution $k_{A}^{*}=\frac{a+b-c+n+1}{c-1}$. We observe that when $c<1, k_{A}^{*}=\frac{a+b-c+n+1}{c-1}$ yields a negative result, which is not a viable solution in the region $c \in[0,1)$ as $k_{A}^{*} \in \mathbb{Z}^{+}$, and hence justifies its exclusion. We now proceed to examine the other solutions at hand for $k_{A}^{*}$.

Here, we would like to remind the reader that $k_{A}^{*}$ is bound by $0 \leq k_{A}^{*} \leq n$. Therefore, solution $k_{A}^{*}=n+1$, is also not feasible as an optimal strategy. In such a case, we argue the boundary conditions $k_{A}^{*}=n$ and $k_{A}^{*}=0$ are the only viable solutions, within $\forall c \in[0, a)$.

Given, there are no solutions for $k_{A}^{*} \in(0, n)$, we conclude that the cost value $c_{n}$ at which $k_{A}^{*}$ starts decreasing from $k_{A}^{*}=$ $n$, and the value $c_{0}$, at which $k_{A}^{*}=0$, both coincide at what we define as the critical point $c_{c}$.

Consequently, the region $c \in[0, a)$ is split by the point $c_{c}$, into two sub-regions, $0 \leq c<c_{c}$ where $k_{A}^{*}=n$ and $c_{c} \leq c<a$ where $k_{A}^{*}=0$. To determine this critical point $c_{c}$, we take a look at the expressions for $u_{A, a v g_{k=n}}$ when $k_{A}^{*}=n$ and $u_{A, a v g_{k=0}}$ for $k_{A}^{*}=0$. We further argue that, at point $c=c_{c}, u_{A, a v g_{k=n}}$ and $u_{A, a v g_{k=0}}$ intersect, as the optimal solution changes from $k_{A}^{*}=n$ to $k_{A}^{*}=0$. Hence, equating $u_{A, a v g_{k=n}}=u_{A, a v g_{k=0}}$ and solving for $c$ we obtain the critical point $c_{c}=-\frac{2 \chi(n+1)-\varphi}{n+2}$ where $\chi=\frac{1}{n+2}\left(\sqrt{(a+b+n)(n+2)+1}+(n+1)^{2}\right)$ and $\varphi=$ $\left(a+b+2(n+1)^{2}\right)$.
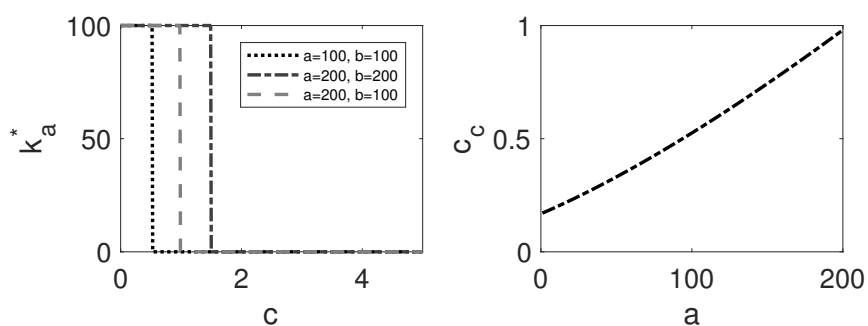

Fig. 2: (a) Plot showing optimal solution for $k_{A}^{*}$ as cost $c$ varies, for different combinations of resources $a$ and $b$, on a star network of $n=100$ nodes. (b) Shows how the critical point $c_{c}$ increases as resource $a$ increases for a fixed $b=100$.

Results shown in this section are particularly exciting as they present an all or none strategy for targeting the peripheral nodes. This produces a typical case, where the system changes from the continuous to discrete models as cost $c$ changes. Fig. 2 further illustrates this abrupt switch from the continuous model $\left(k_{A}^{*}=n\right)$ to the discrete case $\left(k_{A}^{*}=0\right)$, when cost $c$ reaches $c_{c}$. We also observe how this value $c_{c}$ increases as 


\section{IEEE/ACM International Conference on Advances in Social Networks Analysis and Mining}

the available resource $a$ increases, keeping adversarial budget constant.

\section{Adversary targets periphery}

Lemma V.3. The best response strategy against an adversary targeting all peripheral nodes, with a cost of access $\mathrm{c}$, is given by a decreasing function in $c$. When $c \approx 0, k_{A}^{*}=n$, however, as c increases, $k_{A}^{*}$ starts for values $c \geq c_{n}$ and reaches $k_{A}^{*}=0$ at the value $c=c_{0}$.

Proof. In this problem, $\boldsymbol{p}_{\boldsymbol{B}}$ is defined as $p_{B, 0}=0$ and $p_{B, 0}=$ $\frac{b}{n} \forall i$, such that $1 \leq i \leq n$. Keeping $L$ and $p_{A}$ the same as before, we derive the expression for $u_{A, a v g}$. We differentiate $u_{A, \text { avg }}$ wrt $k_{A}$ and $\alpha$ and equate them both to 0 . Upon solving the equations simultaneously, we obtain solutions for $k_{A}^{*}$ and $\alpha^{*}$. For the sake of readability, we refrain from writing down the lengthy expressions obtained for $k_{A}^{*}$ and $\alpha^{*}$.

However, to give the reader an intuition of how the best response strategy varies with change in cost $c$, we plot the closed-form solution for $k_{A}^{*}$ against the cost of accessing nodes $c$ in Fig. 3. for multiple values of resources $a$ and $b$. We observe that as resources $a$ and $b$ increase, in a way that fulfils the condition $a=b$, the respective $c_{n}$ and $c_{0}$ values also increase.

Fig. 3(a) explicitly shows how the $c_{n}$ and $c_{0}$ values increase as resources increase. However, as shown in Fig. 3 (b) it may be worth noting that, $c_{n}$ and $c_{0}$ do not increase in the same proportion, further suggesting that any change in resources has a greater impact on $c_{0}$ than on $c_{n}$. We argue that, as resources increase, it takes longer (or higher cost $c$ ) to change the optimal strategy from targeting some peripheral nodes, to targeting only the hub node.
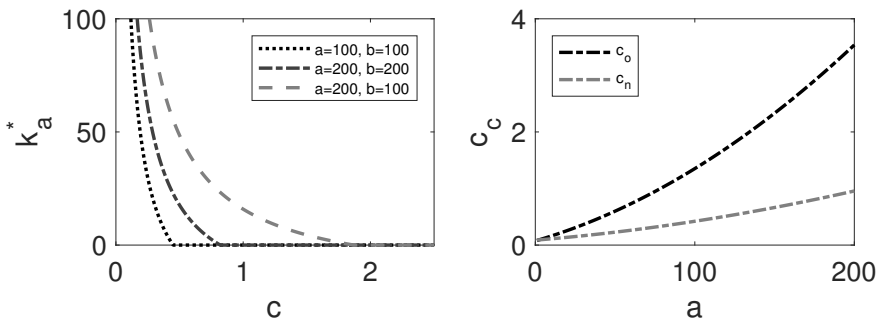

Fig. 3: (a) Plot showing optimal solution for $k_{A}^{*}$ as cost $c$ varies, for different combinations of resources $a$ and $b$, such that $a=b$, on a star network of $n=100$ nodes. (b) Shows how $c_{n}$ and $c_{0}$ increase wrt resources $a$ and $b$.

\section{Game Theoretic ApProACH}

So far we study how the optimal strategy varies with cost of access to nodes $c$ against known adversarial strategies. In this section, we generalise the model further to determine best response strategies against unknown adversarial strategies. Given lack of prior knowledge of the adversarial strategy, we define an arbitrary $\boldsymbol{p}_{B}$ as in Sec. IV to conduct the study further.

$$
\boldsymbol{p}_{\boldsymbol{B}, \boldsymbol{i}}= \begin{cases}b-k_{B} \beta-c\left(k_{B}+1\right), & \text { for } \mathrm{i}=0 \\ \beta, & \text { for } 1 \leq i \leq k_{B} \\ 0, & \text { for } k_{B}+1 \leq i \leq n\end{cases}
$$

As before, values for $L$ and $\boldsymbol{p}_{\boldsymbol{A}}$ remain unchanged from previous sections (Sec. $\mathrm{V}$ ). We can now use Eq. 2 to derive both $u_{A, a v g}$ and $u_{B, a v g}$. Under the current conditions, this yields a complex system that consists of high-order equations that produce inconsistent and unreliable analytical solutions.

We therefore, use a computational method to characterise $N E$ in this setting. Competitors have resources $a$ and $b$ available to them from which they pay a fixed cost $c$ to gain access to the nodes, and a variable cost proportional to the influence exerted on the nodes. Given the current settings, we attempt to understand how the $N E$ changes, with change in cost $c$.

We generate the pay-off matrix numerically by calculating utilities $\left\{u_{A, a v g}, u_{B, a v g}\right\}$ for all available strategies defined by $\left\{\left(k_{A}, \alpha\right),\left(k_{B}, \beta\right)\right\}$. Here $k_{A} \in \mathbb{Z}^{+}$is bounded by $0 \leq$ $k_{A} \leq n$ and is incremented by step-size $\Delta=1$ and $\alpha \in$ $\mathbb{R}^{+}$is incremented by step-size $\Delta=0.01$. Once this matrix has been obtained, we use the Lemke-Howson algorithm [5] to determine the pure or mixed-strategy $N E$ for varying $c$, discretised by $\Delta=0.02$.
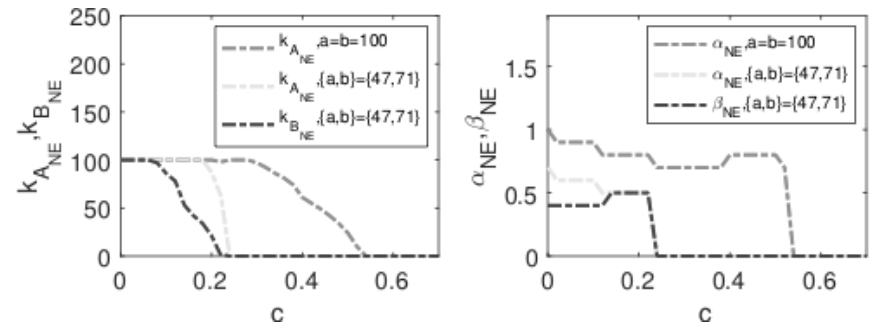

Fig. 4: Plot showing (a) the number of peripheral nodes targeted, (b) the amount of influence expended on the periphery at the $N E$ for different values of $c$, on a star network of $n=100$ nodes.

In Fig. 4, we observe how the $N E$ changes as cost (c) value changes. We also change the values of resources $a$ and $b,(i)$ $\{a, b\}=\{100,100\}$ and an arbitrarily chosen, (ii) $\{a, b\}=$ $\{47,71\}$, to see how it affects the NE. Fig. 4.a), shows how the equilibrium strategy changes in terms of $k_{A_{N E}}$, as $c$ increases, while Fig. 4(b), shows how the amount of influence exerted on the periphery changes with $c$. Note, we only plot one set of equilibrium strategies for the case $a=b$, since both players $A$ and $B$ have the same strategy at $N E$.

We further observe,

1) As cost $c$ increases, the equilibrium strategy gradually shifts from targeting all nodes, to targeting only the hub. This result resembles earlier results, obtained in Sec. IV We also find that, beyond a point $c_{0}$, the strategy $N E$ is always to target the hub node, with all available resources. As resources $a$ and $b$ decrease, it decreases the value of $c_{0}$. 


\section{IEEE/ACM International Conference on Advances in Social Networks Analysis and Mining}

2) Irrespective of the resources available to each player, we see that they both switch their strategy of targeting both hub and peripheral nodes to just the hub node, at the same point $c_{0}$.

\section{CONCLUSION}

In this paper, we explore the $I M$ problem using a dynamic model under competitive settings, where two adversaries compete to spread their influence in the network. Here, nodes are dynamic and are free to continually change their states. We argue this dynamic role, helps us to look at a broader scope of $I M$ problems, in contrast to static models. We also assume that influence is continuously distributed over the network, which contrasts discrete influence in all previous work in this field, adding novelty to our approach.

We consider that influence propagation in our model follows voting dynamics. This allows us to use an analytical framework to study $I M$ in star networks (a prototypical example of the leader-follower structure in social networks) and the role of hub and periphery in best response strategies against both known and unknown adversarial strategies. We observe that as adversarial influence exerted on the network increases, the optimal strategy is to shift resources from nodes targeted by the adversary to those not targeted by the adversary.

Additionally, contrary to previous work [9] [4], our results elicit the importance of peripheral nodes over hub nodes in star networks. Therefore defying the common notion that highdegree nodes are crucial in the $I M$ process.

Lastly, we recognise, that our analytical approach restricts us to simple network structures, such as the star graph. A future direction for this work is to further extend this model to larger networks using optimising algorithms such as Gradient Ascent.

\section{ACKNOWLEDGMENT}

This research was sponsored by the U.S. Army Research Laboratory and the U.K. Ministry of Defence under Agreement Number W911NF-16-3-0001. The views and conclusions contained in this document are those of the authors and should not be interpreted as representing the official policies, either expressed or implied, of the U.S. Army Research Laboratory, the U.S. Government, the U.K. Ministry of Defence or the U.K. Government. The U.S. and U.K. Governments are authorized to reproduce and distribute reprints for Government purposes notwithstanding any copyright notation hereon.

\section{REFERENCES}

[1] Bharathi, S., Kempe, D., \& Salek, M.,2007, December. Competitive influence maximization in social networks. In International workshop on web and internet economics (pp. 306-311). Springer, Berlin, Heidelberg.

[2] Carnes, T., Nagarajan, C., Wild, S. M., \& Van Zuylen, A.,2007, August. Maximizing influence in a competitive social network: a follower's perspective. In Proceedings of the ninth international conference on Electronic commerce (pp. 351-360). ACM.

[3] Kempe, D., Kleinberg, J., \& Tardos, .,2003, August. Maximizing the spread of influence through a social network. In Proceedings of the ninth ACM SIGKDD international conference on Knowledge discovery and data mining (pp. 137-146). ACM.
[4] Kuhlman, C. J., Kumar, V. A., Marathe, M. V., Ravi, S. S., \& Rosenkrantz, D. J.,2010, September. Finding critical nodes for inhibiting diffusion of complex contagions in social networks. In Joint European Conference on Machine Learning and Knowledge Discovery in Databases (pp. 111-127). Springer, Berlin, Heidelberg.

[5] Lemke, C. E., \& Howson, Jr, J. T.,1964. Equilibrium points of bimatrix games. Journal of the Society for industrial and Applied Mathematics, 12(2), 413-423.

[6] Maxwell, K. A.,2002. Friends: The role of peer influence across adolescent risk behaviors. Journal of Youth and adolescence, 31(4), 267-277.

[7] Rogers, E. M.,2010. Diffusion of innovations. Simon and Schuster.

[8] Tichy, N. M., Tushman, M. L., \& Fombrun, C.,1979. Social network analysis for organizations. Academy of management review, 4(4), 507519.

[9] Masuda, N.,2015. Opinion control in complex networks. New Journal of Physics, 17(3), 033031.

[10] Rathnayake, C. and Suthers, D.D., 2016, January. Networked solidarity: An exploratory network perspective on twitter activity related to\# illridewithyou. In 2016 49th Hawaii International Conference on System Sciences (HICSS) (pp. 2058-2067). IEEE.

[11] Wilder, B. and Vorobeychik, Y., 2018, July. Controlling elections through social influence. In Proceedings of the 17th international conference on autonomous agents and multiagent systems (pp. 265-273). International Foundation for Autonomous Agents and Multiagent Systems.

[12] Yildiz, E., Ozdaglar, A., Acemoglu, D., Saberi, A. and Scaglione, A., 2013. Binary opinion dynamics with stubborn agents. ACM Transactions on Economics and Computation (TEAC), 1(4), p.19.

[13] Holley, R.A. and Liggett, T.M., 1975. Ergodic theorems for weakly interacting infinite systems and the voter model. The annals of probability, 3(4), pp.643-663.

[14] Steinert-Threlkeld, Z.C., 2017. Spontaneous collective action: Peripheral mobilization during the Arab Spring. American Political Science Review, 111(2), pp.379-403.

[15] Wu, J.H. and Wang, S.C., 2005. What drives mobile commerce?: An empirical evaluation of the revised technology acceptance model. Information \& management, 42(5), pp.719-729.

[16] Castellano, C., Fortunato, S. and Loreto, V., 2009. Statistical physics of social dynamics. Reviews of modern physics, 81(2), p.591.

[17] He, X., Song, G., Chen, W. and Jiang, Q., 2012, April. Influence blocking maximization in social networks under the competitive linear threshold model. In Proceedings of the 2012 siam international conference on data mining (pp. 463-474). Society for Industrial and Applied Mathematics.

[18] Goyal, S., Heidari, H. and Kearns, M., 2014. Competitive contagion in networks. Games and Economic Behavior.

[19] Anagnostopoulos, A., Ferraioli, D. and Leonardi, S., 2015, May. Competitive influence in social networks: Convergence, submodularity, and competition effects. In Proceedings of the 2015 International Conference on Autonomous Agents and Multiagent Systems (pp. 1767-1768). International Foundation for Autonomous Agents and Multiagent Systems.

[20] Chasparis, G.C. and Shamma, J.S., 2010, December. Control of preferences in social networks. In 49th IEEE Conference on Decision and Control (CDC) (pp. 6651-6656). IEEE.

[21] Fazeli, A., Ajorlou, A. and Jadbabaie, A., 2016. Competitive diffusion in social networks: Quality or seeding?. IEEE Transactions on Control of Network Systems, 4(3), pp.665-675.

[22] Masucci, A.M. and Silva, A., 2014, September. Strategic resource allocation for competitive influence in social networks. In 2014 52nd Annual Allerton Conference on Communication, Control, and Computing (Allerton) (pp. 951-958). IEEE.

[23] Tzoumas, V., Amanatidis, C. and Markakis, E., 2012, December. A game-theoretic analysis of a competitive diffusion process over social networks. In International Workshop on Internet and Network Economics (pp. 1-14). Springer, Berlin, Heidelberg.

[24] Clark, A. and Poovendran, R., 2011, November. Maximizing influence in competitive environments: A game-theoretic approach. In International Conference on Decision and Game Theory for Security (pp. 151-162). Springer, Berlin, Heidelberg.

[25] Wilder, B., Suen, S.C. and Tambe, M., 2018, April. Preventing infectious disease in dynamic populations under uncertainty. In Thirty-Second AAAI Conference on Artificial Intelligence. 